\title{
Tratamiento de la leucemia linfoide aguda del adulto. Experiencia de un hospital en la Ciudad de México
}

\author{
CHRISTIAN RAMOS ${ }^{1}$, ETTA ROZEN ${ }^{1}$, MARÍA LEÓN ${ }^{1}$, \\ ADOLFO MARTÍNEZ T. ${ }^{2}$, IRMA OLARTE², HUMBERTO CATELLANOS ${ }^{1}$, \\ CARLOS MARTÍNEZ ${ }^{1}$, EFREEN MONTAÑO ${ }^{1}$, JUAN KASSACK I. ${ }^{1}$, \\ JORGE ZAMORA², ENRIQUE MIRANDA², ANDRÉS DE LA ROS ${ }^{1}$, \\ MARIO GUTIÉRREZ ${ }^{1}$, JUAN COLLAZO ${ }^{1}$
}

\section{Results of treatment of acute lymphoblastic leukemia in two cohorts of Mexican patients}

Background: GIMEMA ALL 0288 trial was designed to evaluate the impact of a 7-day prednisone (PDN) pretreatment on complete remission of acute lymphoblastic leukemia. We adopted this trial in 2007. Aim: To evaluate the results of treatment in two cohorts of patients with acute lymphoblastic leukemia, treated from 2007 to January 2009 and from February to December 2009. Material and Methods: We studied 99 patients treated in the first period (58 males) and 54 patients treated in the second period (33 males) The age of patients ranged from 16 to 60 years and $70 \%$ of patients were of high risk. BCR/ABL fusion transcript was present in $12 \%$ of patients. Results: Remission rates were 61 and $51 \%$ for patients of the first and second group of treatment, respectively. The main cause of death were infections during the induction period. There were 49 relapses, mainly detected in the blood marrow. Global and event free 34 months survival were 32 and 30\% respectively. Multivariate analysis disclosed risk stratification and central nervous system infiltration as risk factors for mortality. Conclusions: The main obstacles for the treatment of acute lymphoblastic leukemia in these cohorts of patients were the high incidence of infections and the lack of use of growth stimulating factors.

(Rev Med Chile 2011; 139: 1135-1142).

Key words: Daunorubicin; Leukemia, lymphoid; Prednisone.

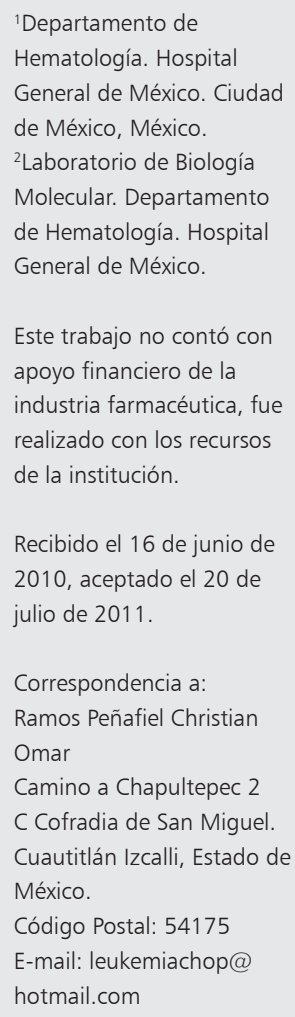

L a leucemia linfoide aguda (LLA) es una enfermedad maligna que se caracteriza por una proliferación descontrolada de células linfoides inmaduras ${ }^{1}$. Su incidencia en México es 5 casos por 100.000 habitantes ${ }^{2}$. La supervivencia en diversas regiones de Latinoamérica oscila entre 24 y $41 \%$ a 5 años $^{3-5}$. En nuestro país, Arteaga y cols en el Instituto Nacional de Ciencias Médicas y Nutrición Salvador Zubirán (INNSZ) han descrito los resultados del protocolo institucional 0195 con una tasa de remisiones completas (RC) del $74 \%$ y una supervivencia global en promedio de 15 meses $^{6}$. En el Hospital General de México desde el año 2007 se estableció un protocolo de tratamiento basado en el protocolo GIMEMA ALL 0288, contando con un ciclo de pre-tratamiento con corticoesteroides semejante a lo establecido en protocolos pediátricos ${ }^{7,8}$. Desde diciembre de 2007 a enero de 2009 se asignó a la administración de daunorrubicina los días $+1,+8,+15$ y en el período de enero de 2009 hasta marzo de 2010 se le asignó la administración de daunorrubicina los días $+1,+2,+3$. Este estudio presenta los resultados del seguimiento a 34 meses y el impacto pronóstico de las diversas variables en el tratamiento de los pacientes portadores de leucemia linfoide aguda atendidos en el departamento de hematología del Hospital General de México. 


\section{Material y Métodos}

\section{Pacientes}

Desde diciembre del 2007 hasta marzo de 2010, se evaluaron a 153 pacientes con diagnóstico de leucemia linfoide aguda de novo que fueron registrados en el Hospital General de México. El diagnóstico se realizó acorde a los criterios morfológicos y se corroboró mediante inmunofenotipo de médula ósea ${ }^{9}$ La detección del transcrito de fusión BCR-ABL se realizó al diagnóstico mediante RT-PCR con oligonucleótidos específicos. La punción lumbar se realizó al inicio del tratamiento.

Entre el período de diciembre de 2007 a enero de 2009 se inició tratamiento acorde al protocolo institucional HGMLAL07 y posteriormente, desde febrero de 2009 se realizó una modificación en la inducción a la remisión reduciendo el intervalo de administración de la daunorrubicina. Ambos protocolos de tratamiento contaban con un pretratamiento en base de una dosis progresiva de esteroides. El esquema de inducción consistió en la administración tanto de alcaloides de la vinca, esteroides y antraciclinas. No se consideró el uso de L-asparaginasa. La profilaxis a sistema nervioso central se realizó con la administración de quimioterapia intratecal semanal durante la inducción y dosis intermedias de metotrexato durante la etapa de consolidación. La duración de la etapa de mantenimiento fue de 2 años con ciclos bimensuales de intensificación con esteroides y alcaloides de la vinca (vincristina). El esquema general de tratamiento se describe en la Tabla 1. Todos los pacientes contaban con el consentimiento informado de la institución al momento de ingreso.

\section{Profilaxis antimicrobiana}

Todos los pacientes se les administro un régimen de profilaxis basado en la administración de fluroquinolonas (ciprofloxacino). El apoyo con factor estimulante de progenitores hematopoyéticos se inició desde el día +5 de tratamiento hasta una cifra de neutrófilos por encima de $0,8 \times 10^{3} / \mu \mathrm{l}$. En caso de aparición de un episodio febril se inicio tratamiento acorde a las recomendaciones internacionales ${ }^{10,11}$.

\section{Clasificación por riesgo y criterios de respuesta}

La estratificación por riesgos se describe en la Tabla 2. Posterior al esquema de inducción a la remisión se consideró $\mathrm{RC}$ a la presencia de $>1 \mathrm{x}$
$10^{3} / \mu \mathrm{L}$ neutrófilos, $>100 \times 10^{3} / \mu \mathrm{L}$ plaquetas y la ausencia de blastos en sangre periférica con menos de $5 \%$ de blastos en médula ósea. Los pacientes los cuales contaron con más de $5 \%$ de blastos posterior a la inducción se les inició un ciclo alternativo de quimioterapia; si no lograron obtener RC se les consideró leucemias refractarias. La clasificación de infiltración a sistema nervioso central se describe en la Tabla 3.

\section{Reacción en cadena de la polimerasa (PCR)}

Se obtuvieron células mononucleares a partir de las muestras de medula ósea, se aisló el ARN celular total por medio de trizol. A partir de $1 \mu \mathrm{g}$ de ARN fue sintetizado el cADN por adición de $1 \mu \mathrm{l}$ de oligo dT, buffer de reacción (200 mM Tris $\mathrm{HCl}$, pH 8,4, $\left.500 \mathrm{mM} \mathrm{KCl}, 25 \mathrm{mM} \mathrm{MgCl}_{2}\right), 1 \mu \mathrm{lde}$ la mezcla de dNTP, $10 \mathrm{mM}$ y $1 \mu \mathrm{l}$ de supertranscriptasa (200 U/ul) (Gibco BRL). Los perfiles térmicos de la reacción fueron a $37^{\circ} \mathrm{C}$ por 50 minutos para la realización de cADN y $70^{\circ} \mathrm{C}$ por 10 minutos para desactivar la enzima supertranscriptasa. El cADN fue almacenado a $-20^{\circ} \mathrm{C}$ hasta su uso.

Se empleó un décimo de cADN para llevar a cabo el análisis de la expresión de los genes $\mathrm{BCR} / \mathrm{ABL}$ en una mezcla de reacción que contenía 30 pMol de cada oligonucleótido sentido 5’TGGAGCTGCAGATGCTGACCAACTCG3'y antisentido ATCTCCACTGGCCACAAAATCATACA3' y 2,5 U de Taq ADN polimerasa (Gibco BRL) en el buffer ( $50 \mathrm{mM} \mathrm{KCl}, 10 \mathrm{mM}$ Tris- $\mathrm{HCl}$ pH 9,0, 0,1\% Triton X-100), 1,5 mM $\mathrm{MgCl}_{2} \mathrm{y}$ $200 \mathrm{uM}$ de cada dNTP. El fragmento esperado amplificado fue de $200 \mathrm{pb}$. Los productos de PCR se visualizado en un gel de agarosa al 1,5\% teñido con bromuro de etidio en un transiluminador de luz UV.

\section{Análisis estadístico}

La supervivencia global y la supervivencia libre de enfermedad se analizaron por el método de Kaplan-Meier y se definieron como el tiempo transcurrido desde el inicio del tratamiento hasta la última fecha registrada (muerte, última fecha de visita) y el tiempo existente entre la remisión completa y la fecha de recaída o última fecha registrada respectivamente. Las diversas variables se analizaron por el método de $\chi^{2}$ y se consideraron significativas si contaban con una $\mathrm{p} \geq 0,050$. 
Tabla 1. Protocolo institucional para el tratamiento de la leucemia linfoide del adulto

\begin{tabular}{|c|c|c|c|c|}
\hline & & & HGMLAL07 & HGMLAL09 \\
\hline Inducción a la r & isión (Fase & e I) & & \\
\hline Daunorrubicina & $60 \mathrm{mg} / \mathrm{m}^{2}$ & IV & $1,8,15$ & $1,2,3$ \\
\hline Vincristina & $1,5 \mathrm{mg} / \mathrm{m}^{2}$ & IV & $, 15,22$ & $1,8,15,22$ \\
\hline Prednisona & $60 \mathrm{mg} / \mathrm{m}^{2}$ & IV & $1-28$ & $1-28$ \\
\hline Citarabina & $40 \mathrm{mg}$ & IT & 15,22 & $1,8,15,22$ \\
\hline Dexa & $8 \mathrm{mg}$ & IT & 2 & $1,8,15,22$ \\
\hline Metotrexatc & & IT & & 5,22 \\
\hline Inducció & ión ( & (I) & & \\
\hline Ciclofosfamida & $50 \mathrm{~m}$ & IV & 1,8 & 1,8 \\
\hline Citar & $65 \mathrm{mg} / \mathrm{m}^{2}$ & IV & $1-4,8$ & $1-4,8$ \\
\hline 6- mercaptopurina & $50 \mathrm{mg} / \mathrm{m}^{2}$ & VO & $1-15$ & $1-15$ \\
\hline Consolidación I & & & & \\
\hline Metotrexat & $1,5 \mathrm{gr} / \mathrm{m}^{2}$ & & $1,15,45$ & $1,15,45$ \\
\hline Intensific & & & & \\
\hline Doxo & $30 \mathrm{mg} / \mathrm{m}^{2}$ & IV & $1,8,15$ & $1,8,15$ \\
\hline Vincristina & $1,5 \mathrm{mg} / \mathrm{m}^{2}$ & IV & $1,8,15,22$ & $1,8,15,22$ \\
\hline Prednisona & $60 \mathrm{mg} / \mathrm{m}^{2}$ & VO & $1-28$ & $1-28$ \\
\hline Consolida & & & & \\
\hline Etoposido & $100 \mathrm{mg} / \mathrm{m}^{2}$ & IV & 1-5, & $1-5$ \\
\hline Citarabina & $75 \mathrm{mg} / \mathrm{m}^{2}$ & IV & $1-5,28-32$ & $1-5,28-32$ \\
\hline Mantenin & อีกต) & & & \\
\hline 6-mercaptopurina & $50 \mathrm{mg} / \mathrm{m}$ & VO & Lune & Lune \\
\hline Metotrexato & $50 \mathrm{mg}$ & $\mathrm{IM}$ & Semanal & Semanal \\
\hline
\end{tabular}

Superficie corporal: $\mathrm{m}^{2}$, VO: vía oral, IM: intramuscular, IV: Intravenoso, IT: intratecal. Al final de cada ciclo de quimioterapia se administró quimioterapia intratecal, durante el mantenimiento se administró cada 2 meses. La médula ósea por aspiración se realizó al final de cada ciclo de quimioterapia.

Tabla 2. Criterios de riesgo

\begin{tabular}{|lcl|}
\hline & Riesgo habitual & Riesgo alto \\
\hline Edad (años) & $<35$ & $>35$ \\
Leucocitos $\left(\times 10^{3} / \mu \mathrm{l}\right)(\mathrm{B})$ & $<30$ & $>30$ \\
Leucocitos $\left(\times 10^{3} / \mu \mathrm{l}\right)(\mathrm{T})$ & $<100$ & $>100$ \\
Remisión completa & $<4$ semanas & $>4$ semanas \\
Líquido cefalorraquídeo* & Negativo & Positivo \\
Inmunofenotipo & B-común & B-madura \\
BCR/ABL & Negativo & T Positivo \\
\hline
\end{tabular}

*Positivo a infiltración a sistema nervioso central.

\section{Resultados}

Desde diciembre de 2007 a febrero de 2010, se analizaron 153 pacientes con el diagnóstico de LAL de novo. La mediana de edad fue de 29 años (rango de 16 a 60 años). Alrededor de 99 pacientes iniciaron tratamiento en el protocolo HGMLAL07 y 54 en el protocolo HGMLAL09. De forma general, $60 \%$ era menor a 35 años y $70 \%$ se clasificó como riesgo alto. El transcrito de fusión BCR/ABL fue positivo en $12 \%$. Debido a la falta de desarrollo en los cultivos de citogenética se consideró el estudio molecular como la técnica de elección para la detección del transcrito de fusión BCR/ABL. Las diferencias entre la población de los dos períodos de estudio se describe en la Tabla 4.

\section{Inducción a la remisión}

De forma general el porcentaje de RC fue de $63 \%$, con una mortalidad en inducción de $26 \%$ y un alrededor de $11 \%$ de leucemias refractarias. De forma individual, la tasa de RC fueron de $60,6 \%$ y $50,5 \%$ para el protocolo HGMLAL07 y HGMLAL09 respectivamente. La principal causa de muerte en inducción fueron los procesos infecciosos asociados a neutropenia febril siendo los principales agentes causales las bacterias Gram-negativas, seguidas de los hongos. La toxicidad hematológica fue el principal evento adverso, la toxicidad hepática sólo se observó en 23 pacientes, 21 de los cuales correspondieron a grado I/II. La recuperación de la cuenta de neutrófilos y plaquetas se observó en 36 y 38 días respectivamente.

Tabla 3. Clasificación alteración en el LCR en la LLA

\begin{tabular}{|ll|}
\hline CNS 1 & Punción lumbar no traumática, menos de 10 eritrocitos $\mathrm{x} \mu \mathrm{l}$ y no blastos identificados por centrifugación \\
CNS 2 & Punción lumbar con menos de 5 leucocitos por $\mu$ l con blastos posterior a la centrifugación \\
CNS 3 & Punción lumbar con punción no traumática con más de 5 leucocitos $\mu$ l con blastos \\
\hline
\end{tabular}

PL traumática Punción lumbar traumática a aquella con más de 10 eritrocitos por microlitro. 
Tabla 4. Características de los pacientes

\begin{tabular}{|c|c|c|}
\hline & HGMLAL07 & HGMLAL09 \\
\hline $\begin{array}{ll}\text { No pacientes } & \\
\text { Masculino } & (\%) \\
\text { Femenino } & (\%) \\
<35 \text { años } & (\%) \\
>35 \text { años } & (\%) \\
\text { BCR/ABL (+) / } 64 \text { pruebas } \\
\text { Infiltración a SNC } / 87 \text { pruebas }\end{array}$ & $\begin{aligned} & \mathrm{N}=99 \\
& 58(58,5) \\
& 41(41,5) \\
& 62(62,6) \\
& 37(37,4) \\
& 9(14 \%) \\
& 2(2,3 \%)\end{aligned}$ & $\begin{aligned} \mathrm{N} & =54 \\
33 & (61,1) \\
21 & (38,9) \\
31 & (57,4) \\
23 & (42,6) \\
3 & (8,8) \\
0 & (0)\end{aligned}$ \\
\hline $\begin{array}{l}\text { Inmunofenotipo / } 74 \text { pruebas } \\
\text { Inmunofenotipo B (\%) } \\
\text { Inmunofenotipo T }(\%)\end{array}$ & $\begin{array}{rr}67 & (90,5) \\
7 & (9,5)\end{array}$ & $\begin{array}{rr}39 & (100) \\
0 & (0)\end{array}$ \\
\hline $\begin{array}{l}\text { Leucocitos } \\
\text { Mediana }\end{array}$ & 39,9 & 39 \\
\hline $\begin{array}{ll}\text { Tipo de riesgo } & \\
\text { Habitual } & (\%) \\
\text { Alto } & (\%)\end{array}$ & $\begin{array}{ll}24 & (24,2) \\
75 & (75,8)\end{array}$ & $\begin{array}{ll}22 & (40,7) \\
32 & (59,3)\end{array}$ \\
\hline
\end{tabular}

\section{Recaída y trasplante de progenitores hematopoyéticos}

Se registraron 48 recaídas siendo la médula ósea el principal sitio, seguido del sistema nervioso central (98\% versus $2 \%$ ) con un tiempo promedio de recaída de 198 días. Dentro de los tratamientos de segunda línea se incluyeron el esquema IDAFLAG, Hyper-CVAD y el protocolo institucional "235". Sólo dos pacientes lograron enviarse en primera remisión a una unidad de trasplante de progenitores hematopoyéticos.

\section{Mortalidad}

Se registraron 65 muertes durante el período de estudio. La etapa donde se registró una mayor tasa de mortalidad fue en inducción con $20 \%$ de todas las defunciones. Entre las principales causas de muerte se encontraron los procesos infecciosos (80\%) y las manifestaciones hemorrágicas (10\%). Los principales agentes infecciosos encontrados en los pacientes con neutropenia febril fueron los Gram-negativos (E. coli) seguido de Sthapylococcus aureus, Sthapyloccoccus epidermidis y candida.

\section{Impacto de los pronósticos en la supervivencia global}

Se observó una diferencia estadísticamente significativa $(\mathrm{p}>0,28)$ para la tasa de $\mathrm{RC}$, pero sin apreciarse una diferencia en cuanto a la supervivencia general (Figura 1) y la supervivencia libre de evento (Figura 2) a 34 meses entre ambos protocolos (HGMLAL07 versus HGMLAL09) $(\mathrm{p}=0,750)$. El inmunofenotipo (lineaje B versus $\mathrm{T})(\mathrm{p}=0,270)$ al igual que la cuenta inicial de leucocitos no mostraron un impacto significativo sobre la supervivencia $(p=0,773)$. En conjunto el tipo de riesgo $(p>0,024)$ al igual que la infiltración al sistema nervioso central mostraron impacto sobre la supervivencia $(\mathrm{p}>0,000)$.

Los resultados del análisis multivariado se describen en la Tabla 5.

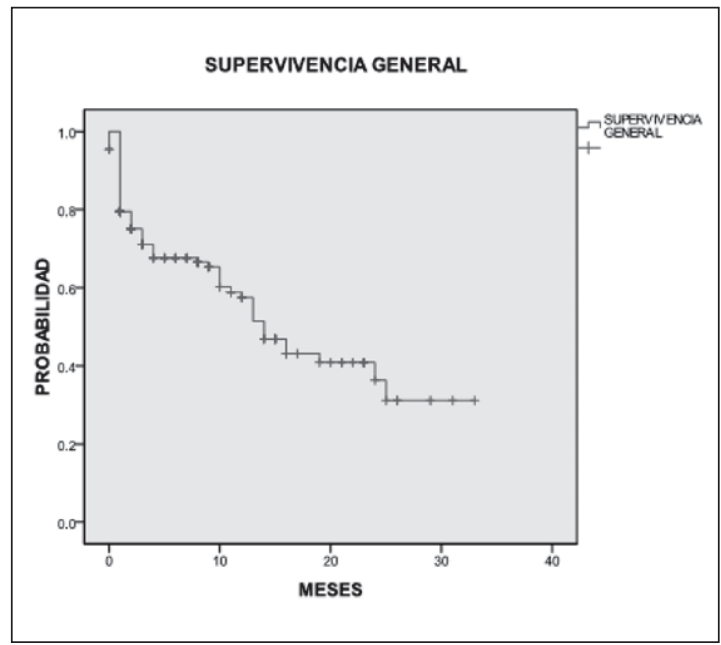

Figura 1. Supervivencia global estimada por el método de Kaplan-Meier a 34 meses de seguimiento. 


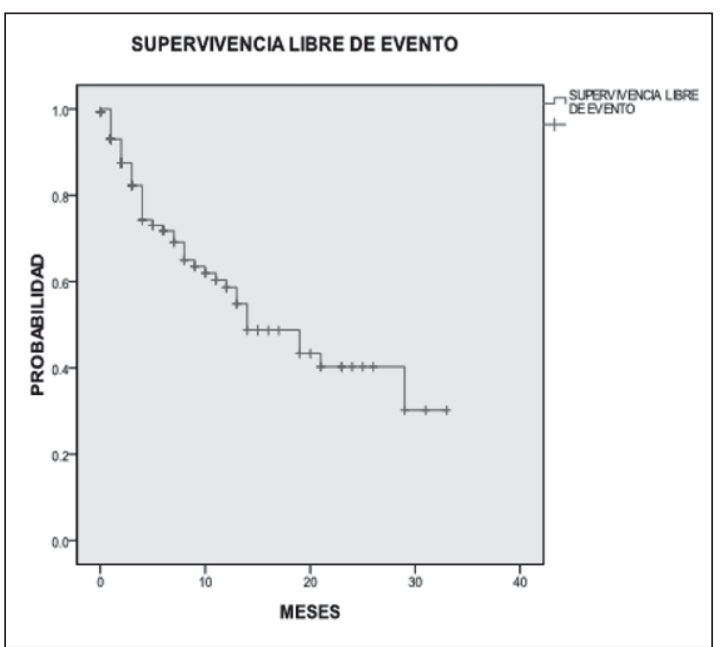

Figura 2. Supervivencia libre de evento estimada por el método de Kaplan-Meier.

Tabla 5. Impacto pronóstico de las diferentes variables de estudio

\begin{tabular}{|lc|}
\hline Variable & $\boldsymbol{p}=$ \\
\hline Riesgo habitual versus Riesgo alto & $\mathrm{p}>0,024$ \\
Cuenta de leucocitos $>35 \times 10^{9} / 1$ & $\mathrm{p}=0,773$ \\
Infiltración a sistema nervioso central & $\mathrm{p}>0,000$ \\
Lineaje B versus lineaje T & $\mathrm{p}=0,270$ \\
BCR/ABL positivo & $\mathrm{p}>0,030$ \\
\hline
\end{tabular}

\section{Pacientes Cromosoma Philadelphia positivos (Ph+)}

Alrededor de $12 \%$ de los pacientes $(\mathrm{n}=18)$ mostraron positividad para el transcrito de fusión BCR/ABL, su presencia mostró impactar estadísticamente sobre la supervivencia $(\mathrm{p}=0,030)$.

\section{Discusión}

Este estudio describe una de las experiencias del tratamiento de la leucemia linfoide aguda desde el año 2007. Contrario a lo reportado en la literatura ${ }^{1,9,10}$, la incidencia de leucemia linfoide aguda del adulto es la leucemia aguda más frecuente en nuestro medio. La frecuencia de algunas variables como lo son el transcrito BCR/ABL fue menor a lo reportado en la literatura $(12 \%)^{13-15}$. Semejante a

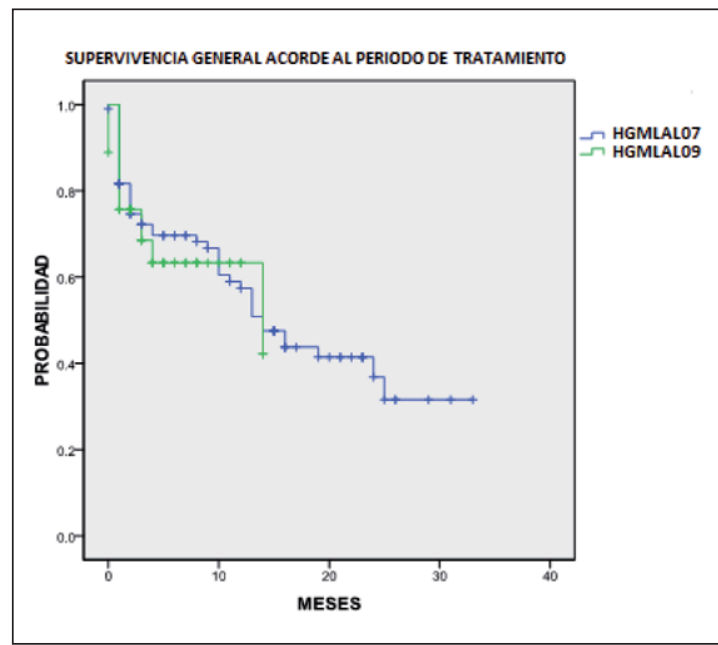

Figura 3. Supervivencia General acorde al período de tratamiento.

otras series internacionales la presencia del transcrito BCR/ABL mostró un impacto significativo en la supervivencia ${ }^{16-18}$. Nuestro protocolo institucional se basa en el protocolo GIMEMA ALL 0288 en donde se realizó un pre-tratamiento con la finalidad de demostrar si la respuesta temprana a esteroides también es un factor pronóstico semejante a lo realizado en series pediátricas ${ }^{19,20}$. En dicho reporte, la mediana de supervivencia fue de 2,2 años ${ }^{7}$ y semejante a otras series como el MRC UKALL XII/ECOG E2993 las variables de riesgo alto mostraron un impacto sobre la superviven$\mathrm{cia}^{21}$. Un punto a considerar de dicho estudio fue la edad, ya que en promedio fue de 27,5 años y hasta $30 \%$ se encontraba en una edad menor a 20 años (12 años como el rango menor). Esta variable pudo influenciar sobre el número de respuesta favorable a esteroides, al igual que en la supervivencia global, ya que ambas son mayores en pacientes pediátri$\cos ^{22}$. Recientemente un sub-análisis de nuestro protocolo institucional HGMLAL07 evidenció que la respuesta favorable a esteroides es baja en el adulto $(27 \%)$ y no impacta sobre la supervivencia genera $^{23}$. La experiencia en Latino-América es diversa, en Argentina, Luesma-Goñalons y cols (GATLA-GLATHEM) evaluaron los resultados del tratamiento de la leucemia linfoide en dos períodos de tiempo (ALL-79, ALL-82) la tasa de RC para el ALL-82 fue 78\% con una supervivencia de $34 \%{ }^{24}$. A diferencia de dicho análisis, nuestro protocolo sustituyó la administración de L- 
aparaginasa por una dosis mayor de antraciclinas (daunorrubcina $25 \mathrm{mg} / \mathrm{m}^{2}$ versus daunorrubina 60 $\mathrm{mg} / \mathrm{m}^{2}$ ) debido a que de forma anecdótica el uso de asparaginasa incrementó los eventos adversos en protocolos previos ${ }^{25}$. Otro punto a considerar en nuestro estudio fue la forma de administración de las antraciclinas. El motivo para considerar acortar el intervalo de dosis fue reducir el tiempo de duración de la aplasia medular con la finalidad de reducir el número de muertes por procesos sépticos. Durante la inducción no se evidenciaron eventos adversos serios cardiovasculares con las dos modalidades de tratamiento, al igual no existió diferencia en el tiempo de duración de la aplasia medular pero si en el porcentaje de remisiones completas (60,6 versus 50,5\%). Semejante a lo sucedido en la experiencia de Puga y cols en Chile, la disposición de factores estimulantes de crecimiento fue limitada siendo un factor significativo en los éxitos de la terapia de inducción ${ }^{26}$. Algunos autores han referido que su administración puede influir en el porcentaje de RC debido a la disminución de los procesos infecciosos severos ${ }^{27}$, pero en general se considera que disminuyen el tiempo de duración de la neutropenia y disminuyen el tiempo de estancia hospitalaria ${ }^{28,29}$. En cuanto al tratamiento de los episodios de neutropenia febril, este se realizó acorde a las consideraciones internacionales. De forma inicial, el tratamiento fue en base a una cefalosporina anti-pseudomona (ceftazidima, cefepime) en combinación con un aminoglucósido (amikacina) en aquellos casos los cuales persistiera el episodio febril o existiera manifestaciones de sepsis se progresaron a un carbapenem (imipenem) en combinación con un aminoglucósido. Sólo en aquellos casos que se presentara inestabilidad hemodinámica o existiera un foco infeccioso ya sea en sitio de venopunción o la sospecha de infección en el acceso infeccioso se inicio la administración de vancomicina ${ }^{30}$. La terapia de escalamiento contó con intervalos de 48 a 72 hrs y se modificó a una terapia específica en aquellos casos los cuales se contara un cultivo positivo. Un punto importante a considerar es que la positividad de los cultivos es baja (menor a $30 \%)^{31}$ y en la mayoría de los pacientes se realizó una terapia empírica. Los principales agentes infecciosos identificados fueron los Gram-negativos, Staphylococcus aureus y en tercer lugar hongos. Esto nos obligó a considerar que la profilaxis antibiótica debe de ser más extensa y actualmente en los protocolos de tratamiento desde abril de 2010 esta se basa tanto en una fluroquinolona (ciprofloxacino), un betalactámico con inhibidor de betalactamasas (amoxicilina/ácido clavulánico), profilaxis antimicótica (fluconazol o itraconazol) y profilaxis contra Pneumocystis jirovecii en base de trimetroprim más sulfametoxazol. Dicha estrategia en conjunto con conceptos obtenidos de las terapias intensivas ${ }^{32}$ han reducido de forma significativa nuestra mortalidad (datos preliminares).

Finalmente, desde hace 20 años no existen modificaciones significativas en el tratamiento de la leucemia linfoide aguda. El régimen de inducción más existoso continúa siendo la combinación de esteroides, alcaloides de la vinca, antraciclinas en algunos protocolos el uso de alquilantes y en los pacientes jóvenes la adición de L-asparaginasa. La profilaxis a sistema nervioso central continúa siendo con la administración intratecal de quimioterapia y el uso de dosis altas o intermedias de metotrexato. Pocos medicamentos se han adicionado desde entonces, algunos de ellos como los inhibidores de cinasa de tirosina de segunda generación (dasatinib) son prometedores en aquellos pacientes $\mathrm{Ph}+$ hasta el momento los estudios fase 2 con hyper-CVAD en conjunto con dasatinib mantienen una supervivencia libre de enfermedad del 64\% a 2 años abriendo el panorama acerca del nuevo papel del trasplante de progenitores hematopoyéticos en este tipo de pacientes ${ }^{33}$. Otros medicamentos como los anticuerpos monoclonales se han estudiado en aquellos pacientes con marcadores como CD20+ y CD19 $+{ }^{34}$. Más recientemente los inhibidores de la vía mTOR (everolimus) se han vuelto una nueva opción en especial en los pacientes con leucemia linfoide $\mathrm{T}^{35}$. A pesar de estos avances alrededor del $60 \%$ de los pacientes presentará una recaída dentro de los primeros 2 años de tratamiento siendo el principal sitio la médula ósea contando con bajas tasas de segundas RC y aun una supervivencia pobre (24 semanas en promedio) ${ }^{36}$. En nuestro reporte, semejante a lo publicado por los grupos internacionales, el principal sitio de recaída fue la médula ósea seguida del sistema nervioso central. Acerca de esto la administración de quimioterapia intratecal con triple droga es eficaz para la prevención de la recaída aislada a sistema nervioso. En conclusión: Este estudio presenta una experiencia latinoamericana en el tratamiento de la leucemia linfoide aguda en el adulta. Dentro de 
los principales obstáculos del tratamiento se encuentran la alta incidencia de procesos infecciosos y la baja disponibilidad a factores estimulantes de crecimiento. Es necesario mejorar las medidas de soporte al igual que implementar estrategias para reducir la mortalidad, principalmente en la etapa de induccion a la remision.

\section{Referencias}

1. Pui CH, Relling M, Downing J. Acute lymphoblastic leukemia. NEJM 2004; 350: 1535-48.

2. Tirado-Gómez L, Mohar Betancourt Mohar A. Epidemiología de las neoplasias hemato-oncológicas. Cancerología 2007; 2: 109-20.

3. Puga B, Cabrera ME. [Results of the National Protocol for Antineoplastic Drugs in the treatment of adult acute lymphoblastic leukemia. Experience at the Hospital del Salvador, 1990-1997]. Rev Med Chile 1998; 126 (9): 1093-9.

4. Rego MF, Pinheiro GS, Metze K, Lorand-Metze I. Acute leukemias in Piauí :comparision with features observed in other regions in Brazil. Braz J Med Biol Res 2003; 36 (3): 331-7.

5. Fogliatto L, Bittencoury H, Nunes AS, Salanave PR, Silva GS, Doudt LE, et al. Outcome of treatment in adult acute lymphoblastic leukemia in southern Brazil a modified german multicenter acute lymphoblastic leukemia protocol. Acta Haematol 2002; 107 (4): 203-7.

6. Arteaga-Ortiz L, Buitrón-Santiago N, Rosas-López A, Rosas-Arzate G, Armengolt-Jiménez A, Aguayo A, et al. Acute lymphoblastic leukemia: experience in adult patients treated with hyperCVAD and 0195 Protocol, at the Instituto Nacional de Ciencias Médicas y Nutrición Salvador Zubirán. Cohort 2003-2007. Rev Invest Clin 2008; 60 (6): 459-69.

7. Annino L, Vegna ML, Camera A, Specchia G, Visani G, Fioritoni G, et al. Treatment of adult acute lymphoblastic leukemia (ALL): long-term follow-up of the GIMEMA ALL 0288 randomized study. Blood 2002; 99: 863-71.

8. Kamps WA, Bökkerink JP, Hählen K, Hermans J, Gadner $\mathrm{H}$, Schrappe M, Slater R, et al. Intensive Treatment of Children With Acute Lymphoblastic Leukemia According to ALL-BFM-86 Without Cranial Radiotherapy: Results of Dutch Childhood Leukemia Study Group Protocol ALL-7 (1988-1991). Blood 1999; 94: 1226-36.

9. Jabbour E, Faderl S. Adult acute lymphoblastic leukemia. Mayo Clinic Proceedings 2005; 80 (11): 1517.

10. Kannangara S. Management of febrile neutropenia. Community Oncology 2006; 3 (9): 585-90.
11. Cullen M, Baijal S. Prvention of febrile neutropenia: use of prophylactic antibiotics. British Journal of Cancer 2009; 101 (S1): S11-14.

12. Gilliland DG, Tallman MS. Focus on acute leukemias. Cancer Cell 2002; 1: 417.

13. Wetzler M, Dodge RK, Mrozek K, Carroll AJ, Tantravahi $\mathrm{R}$, Block AW, et al. Prospective karyotype analysis in adult acute lymphoblastic leukemia: the Cancer and Leukemia Group B experience. Blood 1999; 93: 3983.

14. Mancini M, Scappaticci D, Cimino G, Nanni M, Derme $\mathrm{V}$, Elia L, et al. A comprehensive genetic classification of adult acute lymphoblastic leukemia(ALL):analysis of the GIMEMA 0496 protocol. Blood 2005; 105: 3434-41.

15. Radich J. Philadelphia chromosome-positive acute lymphocytic leukemia. Hematol Oncol Clin North Am 2001; 15 (1): 21.

16. Gleissner B, Gökbuget N, Bartram CR, Janssen B, Rieder $\mathrm{H}$, Janssen JW, et al. Leading prognostic relevance of the BCR-ABL translocation in adult acute B-lineage lymphoblastic leukemia: a prospective study of the German Multicenter Trial Group and confirmed polymerase chain reaction analysis. Blood 2002; 99: 1536-43.

17. Thomas X, Thiebault A, Olteanu N. Philadelphia chromosome positive adult acute lymphoblastic leukemia: characteristics, prognostic factors and treatment outcome. Hematol Cell Ther 1998; 40: 119-28.

18. Barrett AJ, Horowitz MM, Ash RC, Atkinson V, Gale RP, Goldman JM, et al. Bone marrow transplantation for Philadelphia chromosome-positive acute lymphoblastic leukemia. Blood 1992; 79: 3067-70.

19. Schmidt S, Rainer J, Riml S, Ploner C, Jesacher S, Archmüller $\mathrm{C}$, et al. Identification of glucocorticoidresponse genes in children with acute lymphoblastic leukemia. Blood 2006; 107: 2061-9.

20. Aricò M, Valsecchi MG, Conter V, Rizzari C, Pession A, et al. Improved outcome in high risk childhood acute lymphoblastic leukemia defined by prednisone-poor response treated with double Berlin-Frankfurt Muenster protocol II. Blood 2002; 100: 420-6.

21. Fielding AK, Richards S, Chopra R, Lazarus H, Litzow M, Buck G, et al. Outcome of 609 patients after relapse of acute lymphoblastic leukemia; an MRC UKALL12/ ECOG 2993 study. Blood 2007; 109: 944-50.

22. Stanulla M, Scharappe M. Treatment of childhood acute lymphoblastic leukemia. Semin Hematol 2009; 46 (1): 52-63.

23. Ramos C, Castellanos H, Martínez C, Martínez A, Olarte I, Zamora J, et al. Respuesta favorable a esteroides en pacientes con leucemia aguda linfoblástica. Rev Med Hosp Gen Mex 2010; 73 (4): 231-6.

24. Luesma-Goñalons M, Pavlovsky S, Santarelli M, Eppin- 
ger M, Dortticos E, Corrado C, et al. Improved results of an intensified therapy in adult acute lymphocytic leukemia. Annals of Oncology 1991; 2: 33-9.

25. Ramos-Peñafiel CO, Rozen-Fuller E, García-Vidrios V, Rivas-Vera S. Evaluación del tratamiento de la leucemia linfoblástica aguda (Protocolo LAL-2000) en el Hospital General de México: 6 años de seguimiento. Abstract no. 027. Revista de Hematología 2008; 9 (2): S-22.

26. Puga B, Puga I, Cabrera M, Undurraga M, Guerra C, Urrejola G, et al. Neutropenia febril de alto riesgo en leucemia aguda. Experiencia en un hospital público Programa Nacional de Drogas Antineoplásicas del Adulto (PANDA), Hospital del Salvador, 191-2001. Rev Med Chile 2003; 131: 1023-30.

27. Hołowiecki J, Giebel S, Krzemie S, Krawczyk-Kuli M, Jagoda K, Kopera M, et al. G-CSF administered in time-sequenced setting during remission induction and consolidation therapy of adult acute lymphoblastic leukemia has beneficial influence on early recovery and possibly improves long-term outcome: a randomized multicenter study. Leuk Lymphoma 2002; 43 (2): 315-25.

28. Thomas X, Boiron JM, Huguet F, Reman O, Sutton L, Turlure $\mathrm{P}$, et al. Efficacy of granulocyte and granulocytemacrophage colony-stimulating factors in the induction treatment of adult acute lymphoblastic leukemia: a multicenter randomized study. Hematol J 2004; 5 (5): 384-94.

29. Ottmann OG, Bug G, Krauter J. Current status of growth factors in the treatment of acute myeloid and lymphoblastic leukemia. Semin Hematol 2007; 44 (3): 183-92.
30. Penack O, Buchheidt D, Christopeit M, von LilienfeldToal M, Massenkeil G, Hentrich M, et al. Management of sepsis in neutropenic patients: guidelines from the infectious diseases working party of the German Society of Hematology and Oncology. Ann Oncol 2010; 1-9.

31. Syrjälä $H$, Ohtonen P, Kinnunen U, Räty R, Elonen E, Nousiainen T, et al. Blood stream infections during chemotherapy-induced neutropenia in adult patients with acute myeloid leukemia: treatment cycle matters. Eur J Clin Microbiol Infect Dis 2010; 29 (10): 1211-8.

32. Dellinger RP, Carlet JM, Masur H, et al. Surviving sepsis campaign guidelines for management of severe sepsis and septic shock. Crit Care Med 2004; 32: 858-73.

33. Ravandi F, O’Brien S, Thomas D, Faderl S, Jones D, Garris R, et al. First report of phase 2 study of dasatinib with hyper-CVAD for the frontline treatment of patients with Philadelphia chromosome-positive $(\mathrm{Ph}+)$ acute lymphoblastic leukemia. Blood 2010; 116 (12): 2070-7.

34. Thomas DA. Rituximab as therapy for acute lymhoblastic leukemia. Clin Adv Hematol Oncol 2010; 8 (3): 168-71.

35. Vu C, Fruman DA. Target of rapamycin signaling in leukemia and lymphoma. Clin Cancer Res. 2010; 16 (22): 5374-80.

36. Fielding AK, Richards SM, Chopra R, Lazarus HM, Litzow MR, Buck G, et al. Outcome of 609 adults after relapse of acute lymphoblastic leukemia (ALL); an MRC UKALL12/ECOG 2993 study. Blood 2007; 109 (3): 94450. 